\title{
Pressure and Isotropic-Nematic Transition Temperature of Model Liquid Crystals
}

\author{
Siegfried Hess and Bin Su \\ Institut für Theoretische Physik, Technische Universität Berlin, PN 7-1, \\ Hardenbergstr. 36, D-10623 Berlin \\ Reprint requests to Prof. S. H.; Fax: +49 30 31421130, E-mail: S.Hess@physik.tu-berlin.de
}

Z. Naturforsch. 54 a, 559-569 (1999); received June 1, 1999

\begin{abstract}
The pressure in the gaseous, the isotropic liquid and nematic liquid crystalline states, as well as the isotropic-nematic transition temperature are calculated for a model system composed of non-spherical particles. The potential is a generalization of the Lennard-Jones interaction where the attractive part depends on the relative orientations of the particles and the vector joining their centers of mass. Point of departure is an augmented van der Waals approach. It involves a modified Carnahan-Starling expression associated with the repulsive part of the interaction, and an orientation dependent second virial coefficient, as well as the orientational distribution functions of a pair of particles, linked with the attractive part of the potential. In a high temperature approximation, and for a special choice of model parameters, results are presented and displayed graphically.
\end{abstract}

PACS: 05.20.-y, 61.30.by, 64.70.-p, 64.70.Md

Key words: Nematic Liquid Crystal; Interaction Potential; Equation of State.

\section{Introduction}

Recently it has been demonstrated [1] that an augmented van der Waals theory yields good results, over a large density range, for the equation of state of the Lennard-Jones fluid when the short-range repulsive part of the interaction potential (WCA) [2] is taken into account by a modified Carnahan-Starling (CS) equation of state [3, 4]. Reasonable estimates for the gas-liquid coexistence and for the critical temperature can be made. Here this approach is extended to a certain type of model potentials for molecular liquids and liquid crystals. Estimates for the isotropic-nematic transition temperature are given. Though many details are rather different, the theoretical treatment of liquid crystals given here is in the spirit of [5] and [6]. In view of the astonishing variety of chemical compounds showing liquid crystalline phases and the complexity of the meso-phases, the study of new model systems, in addition to the well established ellipsoidal, spherocylinder [7 - 11] and Gay-Berne models, $[12,13]$ is desirable. Due to its simplicity, the present model is well suited for computational studies of the phase behavior of liquid crystals in restricted geometries [14] and of transport processes [15]. The need for the analysis of new models for liquid crystals is also reflected by recent generalizations of the Lebwohl-Lasher [16] lattice model [17, 18].

This article is organized as follows. In Sect. 2, the model potential is introduced. Essentially, it is a generalized Lennard-Jones potential where the $r^{-6}$-part depends on the relative orientations of the axes of the interacting molecules and the vector joining their centers of gravity. Various types of anisotropy of the interaction are taken into account. Then, in Sect. 3, the augmented van der Waals expressions for the free energy and the pressure are presented. An orientation dependent second virial coefficient and the orientational distribution functions of a pair of particles occur in these expressions. The short range repulsive part of the interaction is taken into account by a modified Carnahan-Starling approach. The difference between the free energy in the nematic and isotropic phases, as well as the equilibrium alignment are discussed. Finally, for a special case of the non-spherical interaction and subject to a high temperature approximation, the pressure in the gaseous, in the isotropic liquid and in the nematic liquid crystalline phases, as 
well as the isotropic-nematic transition temperature are computed and displayed graphically in Sect. 4 . Some formulae needed in the calculations are given in the appendix.

\section{The Model}

A fluid composed of (effectively) axisymmetric particles is considered. The binary interaction potential $\Phi$ depends on the relative position vector $r$ joining the centers of mass of two particles and on the unit vectors $\boldsymbol{u}_{1}$ and $\boldsymbol{u}_{2}$ specifying the directions of their figure axes. In general, the potential can be decomposed into an orientationally independent spherical part $\Phi^{\mathrm{sph}}=\Phi^{\mathrm{sph}}(r)$ and a non-spherical or anisotropic part $\Phi^{\text {anis }}=\Phi^{\text {anis }}\left(\boldsymbol{r}, \boldsymbol{u}_{1}, \boldsymbol{u}_{2}\right)$ :

$$
\Phi\left(r, u_{1}, u_{2}\right)=\Phi^{\mathrm{sph}}+\Phi^{\mathrm{anis}} .
$$

The (unconditional) orientational average of $\Phi^{\text {anis }}$ vanishes: $\left\langle\Phi^{\text {anis }}\right\rangle_{0}=0$, where

$$
\langle\ldots\rangle_{0}=(4 \pi)^{-2} \int d^{2} u_{1} \int d^{2} u_{2} \ldots .
$$

As spherical interaction we choose the Lennard-Jones (LJ) potential

$$
\Phi^{\mathrm{LJ}}(r)=4 \Phi_{0}\left(\left(r / r_{0}\right)^{-12}-\left(r / r_{0}\right)^{-6}\right) .
$$

The quantities $\Phi_{0}$ and $r_{0}$ set the characteristic energy and length scales. A characteristic temperature linked with this energy is $k_{\mathrm{B}} T_{\text {ref }}=\Phi_{0}$. For Argon, e.g., one has $T_{\text {ref }}=120 \mathrm{~K}$ and $r_{0}=0.34 \mathrm{~nm}$. The LennardJones (LJ) potential, cut off in its minimum and shifted such that it is zero at the cut-off distance $r_{\text {cut }}$ was used by Weeks, Chandler and Anderson (WCA) [2] as a purely repulsive reference potential. The WCA potential is given by

$$
\begin{aligned}
& \Phi^{\mathrm{WCA}}(r)=4 \Phi_{0}\left(\left(r / r_{0}\right)^{-12}-\left(r / r_{0}\right)^{-6}\right)+\Phi_{0}, \\
& r<r_{\text {cut }}=2^{1 / 6} r_{0} \approx 1.122 r_{0},
\end{aligned}
$$

and $\Phi_{\mathrm{WCA}}(r)=0$ for $r \geq r_{\text {cut }}$.

In numerical calculations and in the graphs displayed here, all physical quantities are expressed in the standard LJ units of [19-25], e.g. lengths and energies are given in units of $r_{0}$ and $\Phi_{0}$. When no danger of confusion exists, the dimensionless variables will be denoted by the same symbols as the original quantities. Then the $\mathrm{LJ}$ and WCA potentials read $\Phi^{\mathrm{LJ}}(r)=4\left(r^{-12}-r^{-6}\right)$, and $\Phi^{\mathrm{WCA}}(r)=$ $4\left(r^{-12}-r^{-6}\right)+1, r<r_{\text {cut }}=2^{1 / 6}, \phi^{\mathrm{WCA}}(r)=0$ for $r \geq r_{\text {cut }}$. The number density $n=N / V$ and the temperature $T$ are in units of $r_{0}^{-3}$ and $\Phi_{0} / k_{\mathrm{B}}$, respectively. The unit for the pressure is $\Phi_{0} r_{0}^{-3}$.

The angle dependence of the anisotropic part of the interaction is described in terms of rotational invariants constructed from irreducible cartesian tensors of rank $\ell$ depending on the components of the unit vectors $\boldsymbol{u}_{1}, \boldsymbol{u}_{2}$ and $\hat{\boldsymbol{r}}=r^{-1} \boldsymbol{r}$. In cartesian component notation, conveniently normalized tensors of rank $\ell=2$ and $\ell=4$ are

$$
\varphi_{\mu \nu}(\boldsymbol{u})=\zeta_{2} \widetilde{u_{\mu} u_{\nu}}, \varphi _ { \mu \nu \lambda \kappa } ( \boldsymbol { u } ) = \zeta _ { 4 } \longdiv { u _ { \mu } u _ { \nu } u _ { \lambda } u _ { \kappa } },
$$

repectively, with $\zeta_{\ell}=\sqrt{(2 \ell+1) ! ! / \ell !}$, in particular

$$
\zeta_{2}=\sqrt{\frac{15}{2}}=\frac{1}{2} \sqrt{30}, \zeta_{4}=\frac{3}{4} \sqrt{70} .
$$

The symbol $\sqcap$ indicates the symetric traceless part of a tensor, e.g. for the dyadic constructed from the components of two vectors $a$ and $b$ one has

$$
\widetilde{a_{\mu} b_{\nu}}=\frac{1}{2}\left(a_{\mu} b_{\nu}+a_{\nu} b_{\mu}\right)-\frac{1}{3} a_{\lambda} b_{\lambda} \delta_{\mu \nu},
$$

where $\delta_{\mu \nu}$ is the unit tensor. The summation convention is used for Greek subscripts. Upon the assumption that the anisotropic part of the potential has a radial dependence proportional to that of the longrange part of the Lennard-Jones potential and taking into account the first five rotational invariants compatible with the head-tail symmetry of nematics, we make the ansatz

$$
\begin{aligned}
\Phi^{\text {anis }} & =-4 \Phi_{0}\left(r / r_{0}\right)^{-6} \Psi\left(u_{1}, u_{2}, \hat{r}\right) \\
\Psi\left(u_{1}, u_{2}, \hat{r}\right) & =\epsilon_{1} \varphi_{\mu \nu}\left(u_{1}\right) \varphi_{\mu \nu}\left(u_{2}\right) \\
& +\epsilon_{2}\left[\varphi_{\mu \nu}\left(u_{1}\right) \varphi_{\mu \nu}(\hat{r})+\varphi_{\mu \nu}\left(u_{2}\right) \varphi_{\mu \nu}(\hat{r})\right] \\
& +\epsilon_{3} \varphi_{\mu \nu}\left(u_{1}\right) \varphi_{\nu \lambda}\left(u_{2}\right) \varphi_{\lambda \mu}(\hat{r}) \\
& +\epsilon_{4} \varphi_{\mu \nu \lambda \kappa}\left(u_{1}\right) \varphi_{\mu \nu \lambda \kappa}\left(u_{2}\right) \\
& +\epsilon_{5} \varphi_{\mu \nu}\left(u_{1}\right) \varphi_{\lambda \kappa}\left(u_{2}\right) \varphi_{\mu \nu \lambda \kappa}(\hat{r})
\end{aligned}
$$

The coefficients $\epsilon_{1, \ldots, 5}$, also refered to as nonsphericity or anisotropy parameters, characterize the 
strenght of the various types of anisotropy considered here. The angle dependent terms in (9) are proportional to the rotational invariants $S^{220}, S^{202}+$ $S^{022}, S^{222}, S^{440}$, and $S^{224}$ used by Stone [26]. Similar tensors and scalar invariants were used in the kinetic theory for gases of particles with spin and scattering theory [27], as well as in the theory of the phase behavior [28] and the calculation of elasticity coefficients of nematics [29]. For generalizations, also to other dimensions, see [30]. Furthermore, notice that one has $\varphi_{\mu \nu}\left(u_{1}\right) \varphi_{\mu \nu}\left(u_{2}\right)=5 P_{2}\left(u_{1} \cdot u_{2}\right)$ where $P_{2}$ is the second Legendre polynomial. The normalization factors $\zeta_{\ell}$ are chosen such that the "square" of the tensors of rank $\ell$ is equal to $2 \ell+1$, e.g. $\varphi_{\mu \nu}(u) \varphi_{\mu \nu}(u)=5$. The Maier-Saupe model [31] corresponds to the case $\epsilon_{1}>0$ and $\epsilon_{2}=\epsilon_{3}=\epsilon_{4}=\epsilon_{5}=0$. For $\boldsymbol{u}_{1}=\boldsymbol{u}_{2}=\boldsymbol{u}$, (9) reduces to $\Psi(\boldsymbol{u}, \boldsymbol{u}, \hat{\boldsymbol{r}})=\psi(\boldsymbol{u}, \hat{\boldsymbol{r}})$ with

$$
\begin{aligned}
\psi(\boldsymbol{u}, \hat{\boldsymbol{r}})= & 5 \epsilon_{1}+9 \epsilon_{4}+\left[2 \epsilon_{2}+\frac{1}{3} \zeta_{2} \epsilon_{3}\right] \varphi_{\mu \nu}(\boldsymbol{u}) \varphi_{\mu \nu}(\hat{r}) \\
& +\zeta_{2}^{2} \zeta_{4}^{-1} \epsilon_{5} \varphi_{\mu \nu \lambda \kappa}(\boldsymbol{u}) \varphi_{\mu \nu \lambda \kappa}(\hat{\boldsymbol{r}})
\end{aligned}
$$

The potential model for perfectly oriented particles ( $\boldsymbol{u}=$ const) presented in [15] and [32] corresponds to (10) with $5 \epsilon_{1}+9 \epsilon_{4}=-1, \epsilon_{5}=0$, and $\epsilon=2 \epsilon_{2}+$ $\frac{1}{3} \zeta_{2} \epsilon_{3}<0$ and $\epsilon>0$ for prolate and oblate particles, respectively. A transition from the nematic to smectic or columnar phases occurs for this special system. The viscous behavior in the vicinity of the phase transition has been studied [15, 32, 33]. Here, we consider fluids with variable orientation and restrict our attention to the isotropic and nematic phases.

The physical meaning of the potential model is obtained by considering the special ee- (end-end), ss- (side-side), T-, and X-configurations corresponding to the cases $\boldsymbol{u}_{1}=\boldsymbol{u}_{2}=\hat{\boldsymbol{r}}, \boldsymbol{u}_{1}=\boldsymbol{u}_{2} \perp \hat{\boldsymbol{r}}$, $\boldsymbol{u}_{1} \perp \boldsymbol{u}_{2}=\hat{\boldsymbol{r}}$, and $\boldsymbol{u}_{1}, \boldsymbol{u}_{2}, \hat{\boldsymbol{r}}$ mutually perpendicular, respectively. In this case, the potential assumes the form

$$
\Phi=4 \Phi_{0}\left(\left(r / r_{0}\right)^{-12}-\left(r / r_{0}\right)^{-6} E_{. .}\right),
$$

with the coefficients $E_{\text {.. }}$ for the main configurations given by

$$
\begin{aligned}
& E_{\mathrm{ee}}=1+5 \epsilon_{1}+10 \epsilon_{2}+\frac{5}{3} \zeta_{2} \epsilon_{3}+9 \epsilon_{4}+\frac{12}{7} \zeta_{4} \epsilon_{5}, \\
& E_{\mathrm{ss}}=1+5 \epsilon_{1}-5 \epsilon_{2}-\frac{5}{6} \zeta_{2} \epsilon_{3}+9 \epsilon_{4}+\frac{9}{14} \zeta_{4} \epsilon_{5},
\end{aligned}
$$
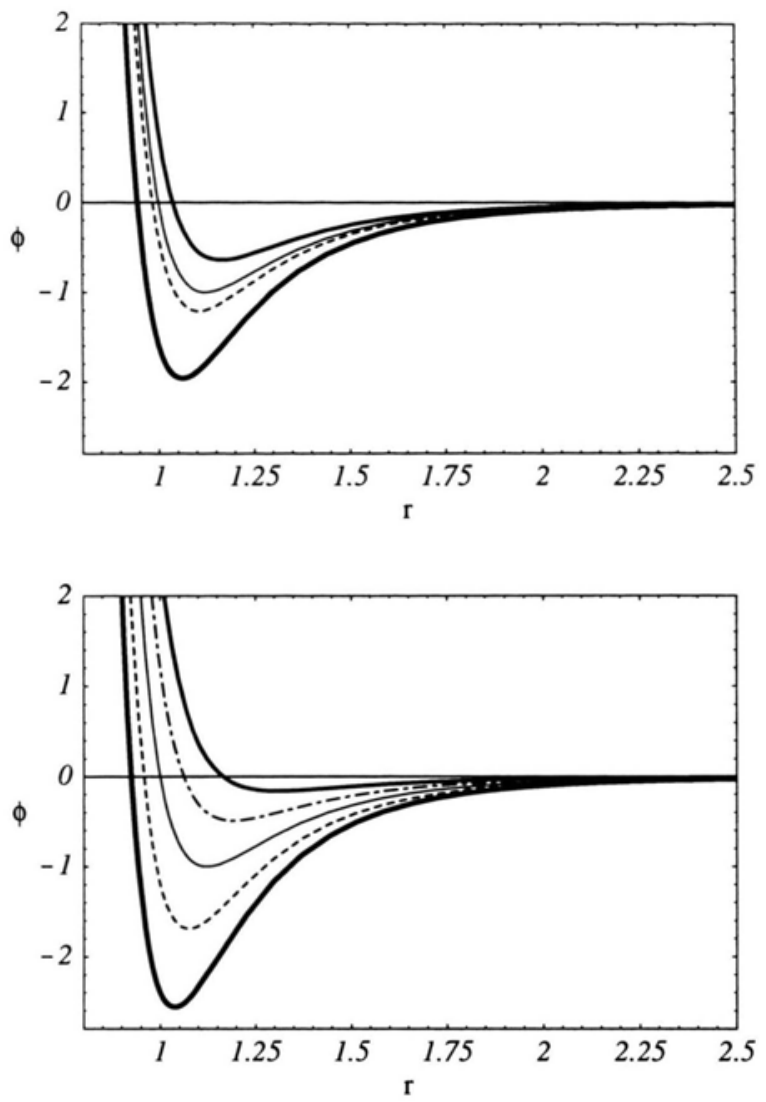

Fig. 1. The potential curves for the principal orientations as functions of the distance $r$. The physical quantities are in standard $\mathrm{LJ}$ units. The top and bottom graphs pertain to $\epsilon_{1}=0.04, \epsilon_{2}=-0.04$, and $\epsilon_{1}=0.04, \epsilon_{2}=-0.08$. The curves with the deepest and shallowest minima correspond to the ss- and ee-orientations, respectively. The thin curve is the spherical Lennard-Jones potential. The dash-dotted and dashed curves are the potential functions for the T- and $\mathrm{X}$-orientations. In the top figure, the $T$-curve coincides with the ee-curve.

$$
\begin{aligned}
& E_{\mathrm{T}}=1-\frac{5}{2} \epsilon_{1}+\frac{5}{2} \epsilon_{2}-\frac{5}{6} \zeta_{2} \epsilon_{3}+\frac{27}{8} \epsilon_{4}-\frac{9}{14} \zeta_{4} \epsilon_{5}, \\
& E_{\mathrm{X}}=1-\frac{5}{2} \epsilon_{1}-5 \epsilon_{2}+\frac{5}{3} \zeta_{2} \epsilon_{3}+\frac{27}{8} \epsilon_{4}-\frac{3}{14} \zeta_{4} \epsilon_{5} .
\end{aligned}
$$

The minimum of the potential is at $r_{\min }=$ $r_{0}\left(2 / E_{. .}\right)^{1 / 6}$, provided that $E_{. .}>0$. The well depth of the potential in the corresponding configuration is $E^{2} \Phi_{0}$.

In the following, the special case $\epsilon_{3}=\epsilon_{4}=\epsilon_{5}=0$ is discussed in more detail. Then one has

$E_{\mathrm{T}}=1-\left(E_{\mathrm{ss}}-1\right) / 2, E_{\mathrm{X}}=1-\left(E_{\mathrm{ee}}-1\right) / 2$. 
For "prolate" particles the side-side configuration is energetically favored over the end-end configuration: $E_{\mathrm{ss}}>E_{\text {ee }}$. This is realized for $\epsilon_{2}<0$. Likewise, oblate particles are modelled by $\epsilon_{2}>0$. The example $E_{\mathrm{ee}}=1 / 2, E_{\mathrm{ss}}=2$, and consequently $E_{\mathrm{T}}=1 / 2$, $E_{\mathrm{X}}=5 / 4$ corresponds to $\epsilon_{1}=1 / 10$ and $\epsilon_{2}=-1 / 10$. Similarly, oblate particles with $E_{\mathrm{ee}}=2, E_{\mathrm{ss}}=1 / 2$, and consequently $E_{\mathrm{T}}=5 / 4, E_{\mathrm{X}}=1 / 2$ pertain to $\epsilon_{1}=$ 0 and $\epsilon_{2}=1 / 10$. The case $\epsilon_{1}=1 / 10$ and $\epsilon_{2}=1 / 10$ leads to oblate particles with $E_{\mathrm{ee}}=5 / 2, E_{\mathrm{ss}}=1$, and consequently $E_{\mathrm{T}}=1, E_{\mathrm{X}}=1 / 4$.

Examples for potential curves with particles in the principal orientations discussed above are displayed in Fig. 1 as functions of the distance $r$. The top and bottom figures pertain to $\epsilon_{1}=0.04, \epsilon_{2}=-0.04$ and $\epsilon_{1}=0.04, \epsilon_{2}=-0.08$. The curves with the deepest and shallowest minima correspond to the side-side (ss) and end-end (ee) orientations, respectively. The thin curve is the spherical Lennard-Jones potential, shown for comparison. The dash-dotted and dashed curves are the potential functions for the $\mathrm{X}$ - and $\mathrm{T}$ orientations. For $\epsilon_{2}=-\epsilon_{1}$, as in the top figure, the T-curve coincides with the ee-curve.

The energetic situations for particles in the principal relative orientations depicted in Fig. 1 can be regarded as characteristic for nematogenic molecules. These molecules, however, have a typical length to width ratio of about 3 which is significantly larger than that one inferred from the positions of the minima of the ee- and ss-potential curves in Figure 1. Thus when one compares with real substances, the non-spherical particle used here should be identified with a small cluster of strongly correlated or associated molecules, packed side by side, rather than a single molecule.

\section{Free Energy and Pressure}

\subsection{General Remarks}

Just as the total internal energy is a sum of the average kinetic and potential energies, most thermomechanical properties are composed of kinetic and potential contributions. The first one is often referred to as ideal gas contribution, the latter ones are sometimes also called excess quantities or configurational contributions. The entropy, and consequently the free energy of nonspherical particles also contains an additional ideal, i.e. single particle contribution, associated with the orientation of the particles.
Let $n=N / V$ be the number density of the fluid of $N$ particles confined to the volume $V$. The free energy $F=F(T, n)=N f(T, n)$, where $f=f(T, n)$ is the free energy per particle, is written as $F=F^{\mathrm{kin}}+F^{\mathrm{or}}+$ $F^{\text {pot }}$, and $f=f^{\text {kin }}+f^{\text {or }}+f^{\text {pot }}$. The kinetic and orientational contributions are $f^{\mathrm{kin}}=k_{\mathrm{B}} T\left(\ln \left(n \lambda^{3}\right)-1\right)$ where $\lambda \sim T^{1 / 2}$ is the thermal de Broglie wave length, and

$$
f^{\text {or }}=k_{\mathrm{B}} T \int \rho \ln \left(\rho / \rho_{0}\right) \mathrm{d}^{2} u .
$$

Here, $\rho=\rho(u)$ is the orientational one-particle distribution function with the normalization $\int \rho \mathrm{d}^{2} u=1$. The random orientation of an isotropic fluid corresponds to $\rho_{0}=(4 \pi)^{-1}$.

The pressure is obtained from the free energy according to

$$
p=n^{2} \frac{\partial f}{\partial n} .
$$

In general, $p$ is a sum of the kinetic contribution $p^{\mathrm{kin}}=$ $n k_{\mathrm{B}} T$ and the potential contribution $p^{\mathrm{pot}}$ associated with $f^{\text {pot }}$ which, in general, depends on the average orientation of the particles.

The potential used here can be viewed as a sum of the short range WCA potential and a long range "distortion" potential

$$
\Phi^{\mathrm{dis}}=\Phi-\Phi^{\mathrm{WCA}} .
$$

The potential contributions to the free energy and pressure can be decomposed accordingly:

$$
f^{\mathrm{pot}}=f^{\mathrm{WCA}}+f^{\mathrm{dis}}, p^{\mathrm{pot}}=p^{\mathrm{WCA}}+p^{\mathrm{dis}} .
$$

For spherical particles, several pertubation schemes have been devised for the calculation of the distortion parts $[2,34,35]$. With a good expression available for the WCA parts, it has been demonstrated that a simple educated guess for $p^{\text {dis }}$ gives surprisingly good results for the pressure of the LJ gas and liquid [1]. Before this approach is generalized to the anisotropic potential functions discussed above, some remarks are made on the WCA reference system.

\subsection{WCA Fluid as Reference System}

For hard spheres with diameter $d$, the CarnahanStarling (CS) expression [4] for the pressure, which 
fits simulation data over the entire density range of the fluid phase rather well, implies $f^{\text {pot }}=f^{\mathrm{CS}}:=$ $k_{\mathrm{B}} T\left\{n B^{h s} /(1-n v)+[n v /(1-n v)]^{2}\right\}$. Here $B^{\mathrm{hs}}=4 v$ and $v=(\pi / 6) d^{3}$ are the second virial coefficient and the volume of a particle. For a fluid of particles with a short-range repulsive interaction $\phi(r)$ like the WCA potential, the CS expression for $f^{\text {pot }}$ is modified by using the virial coefficient $B^{\mathrm{WCA}}=B^{\mathrm{WCA}}(T)$ for the WCA potential. For spherical particles, in general, the second virial coefficient is computed according to

$$
B_{2}(T)=2 \pi \int_{0}^{\infty}\left(1-\exp \left(-\phi(r) / k_{\mathrm{B}} T\right)\right) r^{2} \mathrm{~d} r .
$$

Furthermore, in the modified CS expression, the volume $v$ is replaced by an effective temperaturedependent volume $v_{\text {eff }}(T)$, given by

$$
v_{\text {eff }}(T)=(\pi / 6) d_{\text {eff }}^{3},
$$

with the effective diameter $d_{\text {eff }}=d_{\text {eff }}(T)$ determined by the distance where the binary interaction potential is equal to the thermal energy $k_{\mathrm{B}} T$ :

$$
\phi\left(d_{\text {eff }}\right)=k_{\mathrm{B}} T .
$$

This implies, for the WCA potential,

$$
v_{\text {eff }}(T)=\frac{\pi}{6} r_{0}^{3}\left(2 /\left(1+\left(k_{\mathrm{B}} T / \Phi_{0}\right)^{1 / 2}\right)\right)^{1 / 2} .
$$

Notice that $B^{\mathrm{WCA}} \neq 4 v_{\text {eff }}$, in contradistinction to hard spheres, where one has $B=4 v$. The difference between $B^{\mathrm{WCA}} / 4$ and $v_{\text {eff }}$ is not large but crucial for the quality of the fit for the pressure data [3]. The frequently used recommendation of Barker and Henderson [35] for the computation of an effective diameter yields an effective volume which is just slightly smaller than $B^{\mathrm{WCA}} / 4$ but larger than the $v_{\text {eff }}$ employed here.

The potential contribution to the free energy of the WCA system is

$$
f^{\mathrm{WCA}}=k_{\mathrm{B}} T\left(\frac{n B^{\mathrm{WCA}}(T)}{1-n v_{\mathrm{eff}}(T)}+\left(\frac{n v_{\mathrm{eff}}(T)}{1-n v_{\mathrm{eff}}(T)}\right)^{2}\right) .
$$

The resulting potential contribution to the pressure of the WCA fluid is

$$
p^{\mathrm{WCA}}=n k_{\mathrm{B}} T\left(\frac{n B^{\mathrm{WCA}}}{\left(1-n v_{\mathrm{eff}}\right)^{2}}+2 \frac{\left(n v_{\mathrm{eff}}\right)^{2}}{\left(1-n v_{\mathrm{eff}}\right)^{3}}\right) \text {. }
$$

This expression for the pressure agrees very well with computer simulation data [3], available in the density range $0.1 r_{0}^{-3}<n<1.1 r_{0}^{-3}$ and for the temperatures $T=0.5 T_{\text {ref }}, T_{\text {ref }}, 2 T_{\text {ref }}$, where $T_{\text {ref }}=\Phi_{0} / k_{\mathrm{B}}$. A similar remark applies to the internal energy computed from (25). The densities $n_{\mathrm{fl}}$ and $n_{\text {so }}$ where the fluid phase coexists with the fcc solid phase, at $T=T_{\text {ref }}$, are $n_{\mathrm{fl}}=0.91 r_{0}^{-3}, n_{\mathrm{so}}=0.97 r_{0}^{-3}$.

\subsection{Augmented van der Waals Approximation}

The potential contribution to the pressure of the model system is written as $p^{\text {pot }}=p^{\mathrm{WCA}}+p^{\mathrm{dis}}$. The simplest choice for the distortion part of the pressure is the augmented van der Waals expression [1],

$$
p^{\mathrm{dis}}=k_{\mathrm{B}} T n^{2}\left(B-B^{\mathrm{WCA}}\right),
$$

with the orientationally averaged virial coefficient

$$
B=\iint B\left(\boldsymbol{u}_{1} \cdot \boldsymbol{u}_{2}\right) \rho\left(\boldsymbol{u}_{1}\right) \rho\left(\boldsymbol{u}_{2}\right) \mathrm{d}^{2} u_{1} \mathrm{~d}^{2} u_{2}
$$

and the orientation dependent coefficient

$B\left(\boldsymbol{u}_{1} \cdot \boldsymbol{u}_{2}\right)=\frac{1}{2} \int\left(1-\exp \left(-\Phi\left(\boldsymbol{r}, \boldsymbol{u}_{1}, \boldsymbol{u}_{2}\right) / k_{\mathrm{B}} T\right)\right) \mathrm{d}^{3} r$.

The orientational distribution is written as

$$
\rho(\boldsymbol{u})=\rho_{0}(1+\chi(\boldsymbol{u})), \rho_{0}=(4 \pi)^{-1},
$$

where $\chi(u)$ is a measure for the deviation of $\rho$ from its value $\rho_{0}$ in the isotropic state. The average virial coefficient evaluated for an isotropic state is $B_{\text {iso }}=$ $\langle B\rangle_{0}$. Then (27) can be decomposed into parts which are non-zero in an isotropic state and contribution associated with an average molecular alignment as occurring in the nematic phase:

$$
\begin{aligned}
p^{\mathrm{dis}} & =p_{\text {iso }}^{\mathrm{dis}}+p_{\text {align }}^{\mathrm{dis}} \\
& =k_{\mathrm{B}} T n^{2}\left(B_{\text {iso }}-B^{\mathrm{WCA}}\right)+k_{\mathrm{B}} T n^{2} H
\end{aligned}
$$

with

$$
H=\left\langle\left[B\left(\boldsymbol{u}_{1} \cdot \boldsymbol{u}_{2}\right)-B_{\text {iso }}\right] \chi\left(\boldsymbol{u}_{1}\right) \chi\left(\boldsymbol{u}_{2}\right)\right\rangle_{0} .
$$

This expression for the quantity $H$ is equivalent to

$$
\begin{gathered}
H=-\frac{1}{2}(4 \pi)^{-2} \iiint c_{\text {anis }}\left(\boldsymbol{r}, u_{1}, u_{2}\right) \chi\left(\boldsymbol{u}_{1}\right) \chi\left(\boldsymbol{u}_{2}\right) \\
\cdot \mathrm{d}^{2} u_{1} \mathrm{~d}^{2} u_{2} \mathrm{~d}^{3} r .
\end{gathered}
$$


The anisotropic part of the direct correlation function $c_{\text {anis }}$ here is given by

$$
\begin{aligned}
& -c_{\text {anis }}\left(\boldsymbol{r}, \boldsymbol{u}_{1}, \boldsymbol{u}_{2}\right)= \\
& {\left[\left\langle\exp \left(-\frac{\Phi^{\text {anis }}}{k_{\mathrm{B}} T}\right)\right\rangle_{0}-\exp \left(-\frac{\Phi^{\text {anis }}}{k_{\mathrm{B}} T}\right)\right] \exp \left(-\frac{\Phi^{\text {sph }}}{k_{\mathrm{B}} T}\right) .}
\end{aligned}
$$

As before, $\langle\ldots\rangle_{0}$ indicates an orientational average in an isotropic state.

Equation (27) guarantees the correct behavior of the pressure in the low density limit. However, it is also a good approximation for high densities since the high density behavior is dominated by the WCA part. The pressure $p=n k_{\mathrm{B}} T+p^{\mathrm{WCA}}+p^{\text {dis }}$ with $p^{\text {dis }}$ given by (27) is referred to as the augmented van der Waals equation of state. Notice that one has $B_{\text {iso }}=$ $B_{\text {sph }}+B_{\text {iso }}^{\text {anis }}$ where $B_{\text {sph }}$ is the virial coefficient for the spherical potential, and $B_{\text {iso }}^{\text {anis }}$ is the contribution to the virial coefficient in the isotropic state associated with the anisotropic part of the potential. This quantity is given by

$$
B_{\text {iso }}^{\text {anis }}=\frac{1}{2} \int\left(1-\left\langle\exp \left(-\frac{\Phi^{\text {anis }}}{k_{\mathrm{B}} T}\right)\right\rangle_{0}\right) \exp \left(-\frac{\Phi^{\text {sph }}}{k_{\mathrm{B}} T}\right) \mathrm{d}^{3} r .
$$

\subsection{Free Energy and Equilibrium Alignment}

The distortion part of the free energy pertaining to the pressure (27) is

$$
f^{\text {dis }}=n k_{\mathrm{B}} T\left(B-B^{\mathrm{WCA}}\right)=f_{\text {iso }}^{\text {dis }}+n k_{\mathrm{B}} T H,
$$

in analogy to (31) and with $H$ given by (32) or (33).

The equilibrium alignment of the molecules vanishes in the isotropic phase, but it is finite in the nematic phase. The alignment, in principle, can be computed from the condition $\delta f / \delta \rho(u)=0$, which implies $\ln \rho(\boldsymbol{u}) \sim-2 n \int B\left(\boldsymbol{u} \cdot \boldsymbol{u}_{2}\right) \rho\left(\boldsymbol{u}_{2}\right) \mathrm{d}^{2} u_{2}$. Instead of solving this equation, subject to the normalization condition for $\rho$, one may consider the free energy as a function of the relevant alignment tensors of rank $\ell$ and calculate their equilibrium values by minimizing the free energy. For liquid crystals, the second rank alignment tensor $a_{\mu \nu}$, frequently also denoted by $Q_{\mu \nu}$, plays the role of an order parameter. This tensor is defined by

$$
a_{\mu \nu}=\left\langle\varphi_{\mu \nu}(u)\right\rangle,
$$

where $\langle\ldots\rangle$ indicates an orientational average evaluated with the distribution function $\rho(\boldsymbol{u})$. Higher rank tensors, e.g. of rank $\ell=4,6, \ldots$ are defined analogously. The equilibrium alignment is to be inferred from $\partial f / \partial a_{\mu \nu}=0$, and from similar relations involving the higher rank tensors. The alignment tensors specify the relative deviation $\chi=\left(\rho-\rho_{0}\right) / \rho_{0}$ of the orientational distribution from its isotropic value. More specifically, the expansion with respect to the orthonormalized expansion tensors $\varphi \ldots$ (5) reads

$$
\begin{aligned}
\chi(\boldsymbol{u}) & =\left(\rho-\rho_{0}\right) / \rho_{0} \\
& =a_{\mu \nu} \varphi_{\mu \nu}(\boldsymbol{u})+a_{\mu \nu \lambda \kappa} \varphi_{\mu \nu \lambda \kappa}(\boldsymbol{u})+\ldots .
\end{aligned}
$$

The expansion coefficients are the above mentioned $\ell$-th rank alignment tensors which are defined in analogy to (37). Here we disregard all tensors of rank $\ell \geq 6$. Insertion of the expansion for the distribution function into the expressions for the free energy leads to $f=f_{\text {iso }}+f_{\text {align }}$. The part of the free energy, associated with the alignment (it vanishes in an isotropic state), is given by a Landau-de Gennes type expression [28]

$$
\begin{aligned}
\frac{f_{\text {align }}}{k_{\mathrm{B}} T}= & \frac{1}{2} A_{2} a_{\mu \nu} a_{\mu \nu}-\frac{1}{3} \sqrt{6} B_{0} a_{\mu \nu} a_{\nu \lambda} a_{\lambda \mu} \\
& +\frac{1}{4} C_{0}\left(a_{\mu \nu} a_{\mu \nu}\right)^{2}+\frac{1}{2} A_{4} a_{\mu \nu \lambda \kappa} a_{\mu \nu \lambda \kappa} \\
& -\frac{1}{6} \sqrt{70} D_{0} a_{\mu \nu} a_{\lambda \kappa} a_{\mu \nu \lambda \kappa}+\ldots
\end{aligned}
$$

The coefficients $B_{0}=\sqrt{5} / 7, C_{0}=5 / 7$, and $D_{0}=$ $3 / 7$, already presented in [28], stem from the single particle orientational entropy $f^{\text {or }}$, cf. (17). The coefficients $A_{2}$ and $A_{4}$ also contain contributions involving the interaction of the particles, viz.

$$
\begin{gathered}
A_{2}=1-n \frac{1}{5} \int\left\langle c_{\text {anis }}\left(r, u_{1}, \boldsymbol{u}_{2}\right) \varphi_{\mu \nu}\left(\boldsymbol{u}_{1}\right) \varphi_{\mu \nu}\left(\boldsymbol{u}_{2}\right)\right\rangle_{0} \\
\cdot \mathrm{d}^{3} r
\end{gathered}
$$

and

$$
\begin{gathered}
A_{4}=1-n \frac{1}{9} \int\left\langle c_{\text {anis }}\left(\boldsymbol{r}, \boldsymbol{u}_{1}, \boldsymbol{u}_{2}\right) \varphi_{\mu \nu \lambda \kappa}\left(\boldsymbol{u}_{1}\right) \varphi_{\mu \nu \lambda \kappa}\left(\boldsymbol{u}_{2}\right)\right\rangle_{0} \\
\cdot \mathrm{d}^{3} r
\end{gathered}
$$




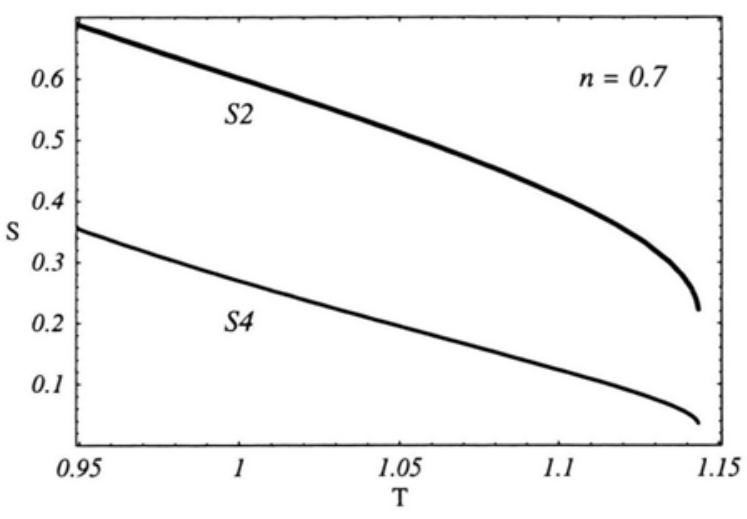

Fig. 2. The order parameters $S_{2}$ and $S_{4}$ as functions of the temperature for the reduced number density $n=0.7$. The anisotropy parameters for the potential function are $\epsilon_{1}=$ $0.04, \epsilon_{2}=-0.08$. The physical quantities are in standard LJ units.

In the nematic phase in equilibrium, the alignment is uniaxial. This means that the alignment tensors are proportional to tensors composed of the components of the (space fixed) unit vector $n$, which is referred to as director. In particular, one has

$$
a_{\mu \nu}=\sqrt{\frac{3}{2}} a_{2} \overline{n_{\mu} n_{\nu}}, a_{\mu \nu} a_{\mu \nu}=a_{2}^{2},
$$

where the magnitude of the second rank tensor is proportional to the Maier-Saupe order parameter $S_{2}=<P_{2}(\boldsymbol{u} \cdot \boldsymbol{n})>$, viz. $a_{2}=\sqrt{5} S_{2}$. Similar relations hold true for the forth rank alignment tensor. Its magnitude $a_{4}$ is linked with $S_{4}=\left\langle P_{4}(\boldsymbol{u} \cdot \boldsymbol{n})\right\rangle$ according to $a_{4}=3 S_{4}$. With this notation, the free energy (39), in a uniaxial state, reduces to [28]

$$
\begin{aligned}
\frac{f_{\text {align }}}{k_{\mathrm{B}} T}= & \frac{1}{2} A_{2} a_{2}^{2}-\frac{1}{3} B_{0} a_{2}^{3}+\frac{1}{4} C_{0} a_{2}^{4}+\frac{1}{2} A_{4} a_{4}^{2} \\
& -D_{0} a_{2}^{2} a_{4}+\ldots
\end{aligned}
$$

Minimization of this alignment free energy leads to $a_{4}=\left(D_{0} / A_{4}\right) a_{2}^{2}$, provided that $A_{4}>0$, and $a_{2}=0$ or $a_{2}=\left(B_{0} / 2 C\right)\left(1+\sqrt{1-4 A_{2} C / B_{0}^{2}}\right)$, depending on whether the temperature and density dependent coefficient $A_{2}$ is larger or smaller than $A_{K}=2 B_{0}^{2} /(9 C)$. Here, the coefficient $C$ is given by $C=C_{0}-2 D_{0}^{2} / A_{4}$. At the temperature $T_{K}$ where the isotropic phase coexists with the nematic phase, the magnitude of the second rank alignment tensor is equal to $a_{K}=2 B_{0} /(3 C)$. This corresponds to $S_{2}=$ $(2 / 15)\left(1-(18 / 35) A_{4}\left(T_{K}\right)^{-1}\right)^{-1}>14 / 51 \approx 0.28$, at the transition temperature. The inequality follows from $A_{4}<1$. The value $27 / 35$ for $A_{4}\left(T_{K}\right)$ implies $S_{2}=0.4$ which is typical for many nematics just below the transition temperature. To provide an example, the order parameters $S_{2}$ and $S_{4}$ are shown in Fig. 2 as functions of the temperature, for the number density $n / n_{\text {ref }}=0.7$. The anisotropy parameters for the potential function are $\epsilon_{1}=0.04, \epsilon_{2}=-0.08$. The curves have been computed in the "high temperature approximation" to be discussed below.

The pseudo-critical temperature $T^{*}$ where one has $A_{2}=0$ is slightly smaller than the transition temperature. The calculation of $T^{*}$, to be presented later, provides a lower bound on the transition temperature. The the pase behavior and the pressure in the various phases are studied next, subject to reasonable and manageable approximations.

\section{Gas, Liquid and Nematic Liquid Crystal}

In order to obtain reasonable estimates for the transition temperature and for the pressure in the isotropic and nematic phases, a "high temperature approximation", with respect to the anisotropic part of the interaction potential, is used in the following. More specifically, $\exp \left[-\Phi^{\text {anis }} / k_{\mathrm{B}} T\right]$ is expanded up to second order in $\Phi^{\text {anis }} / k_{\mathrm{B}} T$; the spherical part of the interaction potential, however, is taken into account in all orders.

\subsection{High Temperature Approximation}

The coefficients $A_{2}$ and $A_{4}$ ocurring in the free energy are computed next, with $c_{\text {anis }}$ replaced by the high temperature approximation:

$$
\begin{aligned}
c_{\text {anis }}^{\text {hit }}\left(r, u_{1}, u_{2}\right)=\left[-\Phi^{\text {anis }}\left(k_{\mathrm{B}} T\right)^{-1}+\frac{1}{2}\left(\left(\Phi^{\text {anis }}\right)^{2}\right.\right. \\
\left.\left.-\left\langle\left(\Phi^{\text {anis }}\right)^{2}\right\rangle_{0}\right)\left(k_{\mathrm{B}} T\right)^{-2}\right] \exp \left(-\Phi^{\text {sph }} / k_{\mathrm{B}} T\right) .
\end{aligned}
$$

Insertion of this expression into (40) and (41), with the anisotropic part of the potential given by (8) and (9) yields

$$
\begin{aligned}
& A_{2}=1-\frac{T_{1}}{T}-\frac{T_{2}^{2}}{T^{2}}=1-\frac{n}{n_{2}}, \\
& A_{4}=1-\frac{T_{4}}{T}-\frac{T_{42}^{2}}{T^{2}}=1-\frac{n}{n_{4}} .
\end{aligned}
$$




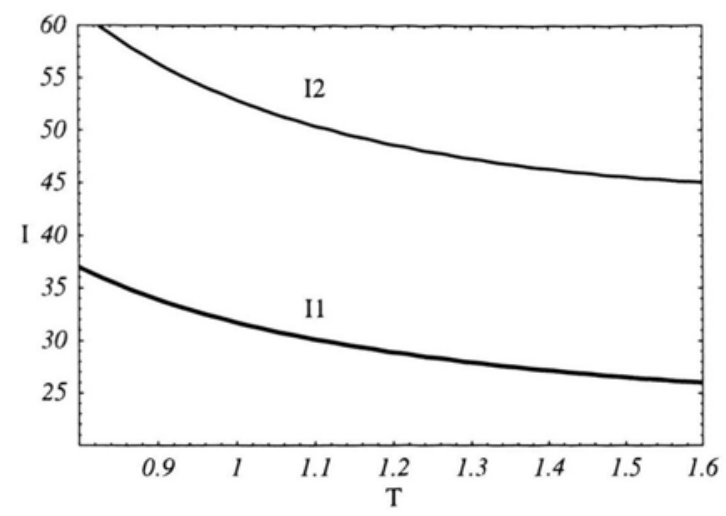

Fig. 3. The integrals $I_{1}$ and $I_{2}$ as functions of the temperature (in reduced units).

The characteristic quantities $T_{1}, T_{2}, T_{4}, T_{42}$, which have the dimension of a temperature, are determined by

$$
\begin{aligned}
k_{\mathrm{B}} T_{1} / \Phi_{0}= & \epsilon_{1} n r_{0}^{3} I_{1},\left(k_{\mathrm{B}} T_{2} / \Phi_{0}\right)^{2}=\epsilon_{22} n r_{0}^{3} I_{2}, \\
\epsilon_{22}= & \frac{5}{7} \epsilon_{1}^{2}+\epsilon_{2}^{2}-\frac{5}{56} \epsilon_{3}^{2}+\frac{2}{3} \zeta_{2} \epsilon_{2} \epsilon_{3} \\
& +\frac{90}{77} \epsilon_{4}^{2}+\frac{18}{49} \epsilon_{5}^{2}+\frac{18}{7} \epsilon_{1} \epsilon_{4},
\end{aligned}
$$

and

$$
\begin{gathered}
k_{\mathrm{B}} T_{4} / \Phi_{0}=\epsilon_{4} n r_{0}^{3} I_{1},\left(k_{\mathrm{B}} T_{42} / \Phi_{0}\right)^{2}=\epsilon_{42} n r_{0}^{3} I_{2}, \\
\epsilon_{42}=\frac{5}{7} \epsilon_{1}^{2}+\frac{5}{42} \epsilon_{3}^{2}+\frac{729}{1001} \epsilon_{4}^{2}+\frac{1}{98} \epsilon_{5}^{2}+\frac{100}{77} \epsilon_{1} \epsilon_{4} .
\end{gathered}
$$

The dimensionless quantities $I_{1}, I_{2}$ are abbreviations for

$$
\begin{aligned}
& I_{1}=4 r_{0}^{-3} \int\left(r / r_{0}\right)^{-6} \exp \left(-\Phi^{\mathrm{LJ}} / k_{\mathrm{B}} T\right) \mathrm{d}^{3} r, \\
& I_{2}=16 r_{0}^{-3} \int\left(r / r_{0}\right)^{-12} \exp \left(-\Phi^{\mathrm{LJ}} / k_{B} T\right) \mathrm{d}^{3} r .
\end{aligned}
$$

The characteristic number densities $n_{2}$ and $n_{4}$ are determined by

$$
\begin{aligned}
& n_{2}=r_{0}^{-3}\left(\left(\Phi_{0} / k_{\mathrm{B}} T\right) \epsilon_{1} I_{1}+\left(\Phi_{0} / k_{\mathrm{B}} T\right)^{2} \epsilon_{22} I_{2}\right)^{-1}, \\
& n_{4}=r_{0}^{-3}\left(\left(\Phi_{0} / k_{\mathrm{B}} T\right) \epsilon_{4} I_{1}+\left(\Phi_{0} / k_{\mathrm{B}} T\right)^{2} \epsilon_{42} I_{2}\right)^{-1} .
\end{aligned}
$$

Formulas given in the appendix have been used. In the following, the consequences of the high temperature approximation are exploited for the special case where $\epsilon_{3}=\epsilon_{4}=\epsilon_{5}=0$. The dependence of the quantities $I_{1}, I_{2}$ on $T$ is shown in Figure 3 .

\subsection{Estimate for the Isotropic-Nematic Transition Temperature and Density}

When the temperature dependence of $T_{1}, T_{2}$ is ignored, the pseudo-critical temperature $T^{*}$ inferred from $A_{2}=0$ is, for $\epsilon_{1}>0$, given by

$$
T^{*}=\frac{1}{2} T_{1}\left(1+\left(1+4 T_{2}^{2} / T_{1}^{2}\right)^{-1 / 2}\right) .
$$

For $\epsilon_{1}=0$, but $\epsilon_{2} \neq 0$ one has $T^{*}=T_{2}$. An order of magnitude estimate of the dependence of $T^{*}$ on $\epsilon_{1}$ and $\epsilon_{2}$ is obtained with $I_{1} \approx 30, I_{2} \approx 50$ (at the reduced temperature $T=1$ one has $I_{1}=31.7$ and $I_{2}=52.9$, cf. Fig. 3), and for $n r_{0}^{3}=0.67$ :

$$
T^{*} \approx 10 \epsilon_{1}\left(1+\left(1+\frac{1}{2}\left(1+\frac{7}{5}\left(\frac{\epsilon_{2}}{\epsilon_{1}^{2}}\right)^{-1 / 2}\right) .\right.\right.
$$

This leads to $T^{*} \approx 22,25,28 \epsilon_{1}$ for $\epsilon_{2} / \epsilon_{1}=$ $0,-1,-2$. Thus the transition temperature is mainly determined by $\epsilon_{1}$. The value for $\epsilon_{1}=0.04$ implies a reduced transition temperature $\approx 1$. A nonzero value of $\epsilon_{2}$ leads to an increase of $T^{*}$. In Fig. 6, the thick line on the right indicates where one has $A_{2}=0$, in the temperature-density plane, for a specific choice of the non-sphericity parameters.

\subsection{Shift of the Critical Temperature}

Of course, the van der Waals theory cannot describe correctly the fluctuation-dominated behavior in the immediate vicinity of the critical point of a real fluid. Nevertheless, estimates of the critical temperature and density, $T_{\mathrm{c}}$ and $n_{\mathrm{c}}$, from mean-field theory are quite useful. The simple augmented van der Waals equation of state with the distortion part of the pressure given by (27) implies $T_{\mathrm{c}} \approx 1.28 T_{\text {ref }}, n_{\mathrm{c}} \approx 0.25 n_{\text {ref }}$, and $p_{\mathrm{c}} /\left(n_{\mathrm{c}} k_{\mathrm{B}} T_{\mathrm{c}}\right) \approx 0.33$ for the LJ fluid.

A shift of $T_{\mathrm{c}}$ due to an anisotropic potential is caused by $B_{\text {iso }}^{\text {anis }} \neq 0$. In the high temperature approximation with respect to the anisotropic part of the interaction, in the spirit of the Barker-Pople expansion $[36,37]$, one obtains

$$
\begin{aligned}
B_{\text {iso }}^{\text {anis }} & \approx-\frac{1}{4} \int\left\langle\left(\Phi^{\text {anis }} / k_{\mathrm{B}} T\right)^{2}\right\rangle_{0} \exp \left(-\Phi^{\mathrm{sph}} / k_{\mathrm{B}} T\right) \mathrm{d}^{3} r \\
& =-\epsilon_{02}\left(\Phi_{0} / k_{\mathrm{B}} T\right)^{2} r_{0}^{3} I_{2}
\end{aligned}
$$



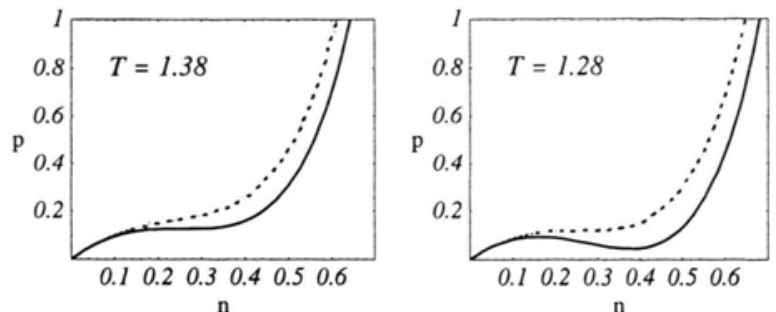

Fig. 4. The pressure in the isotropic state as function of the density for the reduced temperatures $T=1.38$ (left) and 1.28 (right) for the model system with $\epsilon_{1}=0.04, \epsilon_{2}=$ -0.08 . The dashed curves pertain to the Lennard-Jones liquid. The higher temperature is the critical temperature of the model liquid crystal, the lower one is the critical temperature of the $\mathrm{LJ}$-fluid. The physical quantities are in standard LJ units.

with

$\epsilon_{02}=\frac{5}{4} \epsilon_{1}^{2}+\frac{5}{2} \epsilon_{2}^{2}+\frac{35}{48} \epsilon_{3}^{2}+\frac{5}{3} \zeta_{2} \epsilon_{2} \epsilon_{3}+\frac{9}{4} \epsilon_{4}^{2}+\frac{9}{4} \epsilon_{5}^{2}$.

Again, formulas given in the appendix have been used. An estimate for $B_{\text {iso }}^{\text {anis }}$ is given for the special case $\epsilon_{3}=\epsilon_{4}=\epsilon_{5}=0$. With $I_{2} \approx 50$ and $\epsilon_{1}=0.05$, one finds $B_{\text {iso }}^{\text {anis }} / r_{0}^{3} \approx-1 / 6,-1 / 2,-3 / 2$ for $\epsilon_{2} / \epsilon_{1}=0,-1,-2$, respectively. In the expression for the pressure, these numbers for $B_{\text {iso }}^{\text {anis }}$ have to be compared with $B_{\mathrm{sph}}-B^{\mathrm{WCA}} \approx-6 r_{0}^{3}$, for the $\mathrm{LJ}$ potential. Notice that $B_{\text {iso }}^{\text {anis }}<0$ leads to an increase of both the critical temperature and density. Specific examples for pressure curves in the isotropic phase of the model liquid crystal with $\epsilon_{1}=0.04, \epsilon_{1}=-0.08$, are displayed in Fig. 4, together with the pressure for the LJ-fluid (dashed curves). The temperatures have been chosen such that they correspond to the critical temperatures of the liquid crystal and of the LJ-system, viz. $T / T_{\text {ref }}=1.38$, (left) and 1.28 (right), respectively.

\subsection{Pressure in the Isotropic and Nematic Phases}

The present approach allows the calculation of the pressure both in the isotropic and nematic phases. In the first case, the pressure is $p_{\text {iso }}=p^{\mathrm{WCA}}+$ $n^{2} k_{\mathrm{B}} T\left(B_{\mathrm{sph}}-B^{\mathrm{WCA}}\right)+n^{2} k_{\mathrm{B}} T, B_{\text {iso }}^{\text {align }}$, in the second case, one has the pressure $p_{\text {nem }}=p_{\text {iso }}+p_{\text {align }}^{\text {dis }}$ with

$$
p_{\text {align }}^{\mathrm{dis}}=-\frac{1}{2} n k_{\mathrm{B}} T\left(\frac{n}{n_{2}} a_{2}^{2}+\frac{n}{n_{4}} a_{4}^{2}\right) .
$$
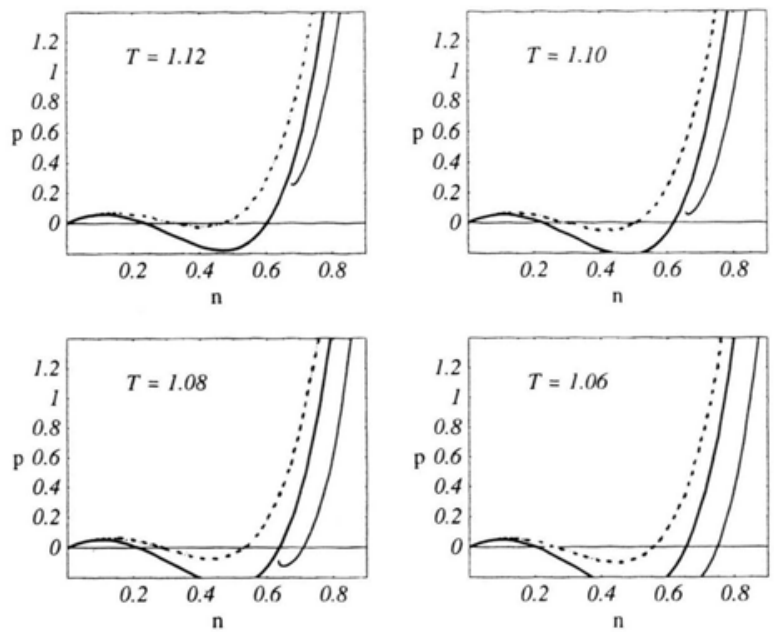

Fig. 5. The pressure in the isotropic and nematic phases as function of the density for the reduced temperatures $T=$ $1.12,1.10$ (top) and $T=1.08,1.06$ (bottom) for the model system with $\epsilon_{1}=0.04, \epsilon_{2}=-0.08$. The dashed curves are for the Lennard-Jones liquid. The physical quantities are in standard LJ units.

In Fig. 5 the pressure curves for the isotropic and the nematic phases are displayed as functions of the density for the temperatures $T / T_{\text {ref }}=1.12,1.10$ (top) and $T / T_{\text {ref }}=1.08,1.06$ (bottom). The model system studied is charcterized by $\epsilon_{1}=0.04, \epsilon_{2}=-0.08$. The dashed curves, shown for comparison, are for the LJ fluid. In all four figures, the outer most curves pertain to the nematic phase. For a given pressure, the density in the nematic phase is higher than in the isotropic phase. Notice that at the higher temperatures (top), the nematic state can only be reached at a finite pressure, whereas at the lower temperatures (bottom), the nematic and isotropic phases can coexist at zero pressure.

\subsection{A Qualitative Phase Diagram}

The limits of stability of the gas and of the isotropic liquid as inferred from the spinodal where one has $\partial p / \partial n=0$, and from $p=0$, are indicated in Fig. 6, in the temperature-density plane, for the model fluid with the non-sphericity parameters $\epsilon_{1}=0.04, \epsilon_{2}=$ -0.08 . The thick line on the right marks the locus where the coefficient $A_{2}$ in the free energy vanishes, which is close to the isotropic-nematic phase transition. Even without a detailed analysis of the free energy which would require a Maxwell construction for coexisting states, the curves shown provide a 


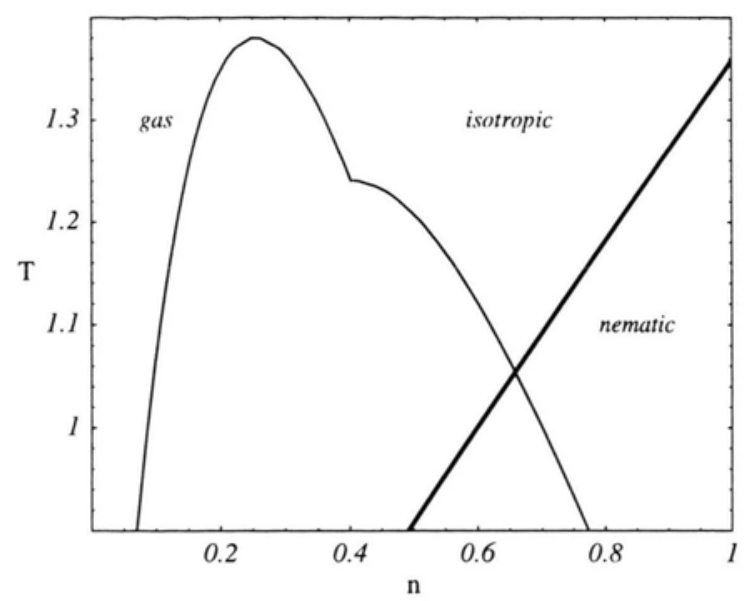

Fig. 6. Phase diagram for the model system with $\epsilon_{1}=$ $0.04, \epsilon_{2}=-0.08$. The thick line on the right marks the pseudo-critical temperature where the coefficient $A_{2}$ vanishes. The other curves indicate the limits of stability in the isotropic phase as infered from the spinodal where $\partial p / \partial n=0(n<0.4)$ and from $p=0(n>0.4)$. The physical quantities are in standard $\mathrm{LJ}$ units.

qualitative phase diagram indicating for which temperatures and densities a gas, an isotropic liquid or a nematic liquid crystalline phase is expected.

\section{Concluding Remarks}

In this article, the augmented van der Waals approach has been extended to a specific type of nonspherical interaction potential. For a special case, explicit expressions have been obtained and displayed graphically for the pressure in the gaseous, in the isotropic liquid and in the nematic liquid crystalline phases, as well as for the isotropic-nematic transition temperature. It is expected that the liquid crystal model considered here also possesses smectic phases. Their possible occurrence, in particular the phase transition nematic-smectic A can be studied by similar methods.

The model potential introduced in Sect. 2 is well suited for numerical studies of equilibrium and nonequilibrium material properties of liquid and liquid crystalline substances, both in bulk and in restricted geometries. The considerations presented here can serve as guide lines for the choice of model parameters and state variables even when the mean field theory, together with the approximations discussed above, has only a limited quantitative accuracy. Of course, also further analytic calculations are desir- able, in particular the computation of the Frank elasticity coefficients and of interfacial properties along the lines indicated in [38].

\section{Acknowledgements}

This work has been performed under the auspices of the Sonderforschungsbereich 448 "Mesoskopisch strukturierte Verbundsysteme". Financial support by the Deutsche Forschungsgemeinschaft is gratefully acknowleged. We thank Martin Schoen, Martin Kröger, Götz Rienäcker and Heiko Steuer for helpful discussions.

\section{Appendix: Directional Averages}

The averages of the quantity $\Psi$, defined in (9), and of $\Psi^{2}$, over the unit vector $\hat{\boldsymbol{r}}$, needed in the previous calculations, are:

$$
\begin{aligned}
& (4 \pi)^{-1} \int \Psi\left(u_{1}, u_{2}, \hat{r}\right) \mathrm{d}^{2} \hat{r}=\epsilon_{1} \varphi_{\mu \nu}\left(u_{1}\right) \varphi_{\mu \nu}\left(u_{2}\right) \\
& \quad+\epsilon_{4} \varphi_{\mu \nu \lambda \kappa}\left(u_{1}\right) \varphi_{\mu \nu \lambda \kappa}\left(u_{2}\right)=\epsilon_{1} 5 P_{2}+\epsilon_{4} 9 P_{4}, \\
& (4 \pi)^{-1} \int\left(\Psi\left(u_{1}, u_{2}, \hat{r}\right)\right)^{2} d^{2} \hat{r}=\epsilon_{1}^{2}\left(\varphi_{\mu \nu}\left(u_{1}\right) \varphi_{\mu \nu}\left(u_{2}\right)\right)^{2} \\
& +2 \epsilon_{2}^{2}\left(5+\varphi_{\mu \nu}\left(u_{1}\right) \varphi_{\mu \nu}\left(u_{2}\right)\right) \\
& +\epsilon_{3}^{2} \varphi_{\mu \nu}\left(u_{1}\right) \varphi_{\nu \lambda}\left(u_{2}\right) \\
& +2 \epsilon_{\lambda \kappa}\left(\epsilon_{3}\right) \varphi_{\kappa \mu}\left(\varphi_{\mu \nu}\left(u_{1}\right)+\varphi_{\mu \nu}\left(u_{2}\right)\right) \varphi_{\mu \lambda}\left(u_{1}\right) \varphi_{\lambda \nu}\left(u_{2}\right) \\
& +\epsilon_{4}^{2}\left(\varphi_{\mu \nu \lambda \kappa}\left(u_{1}\right) \varphi_{\mu \nu \lambda \kappa}\left(u_{2}\right)\right)^{2} \\
& +\epsilon_{5}^{2} \overbrace{\mu \nu}\left(u_{1}\right) \varphi_{\lambda \kappa}\left(u_{2}\right) \\
& +2 \epsilon_{1} \epsilon_{4} \varphi_{\alpha \beta}\left(u_{1}\right) \varphi_{\alpha \beta}\left(u_{2}\right) \varphi_{\mu \nu \lambda \kappa}\left(u_{1}\right) \varphi_{\mu \nu \lambda \kappa}\left(u_{2}\right) \\
& =\epsilon_{1}^{2}\left(5 P_{2}\right)^{2}+10 \epsilon_{2}^{2}\left(1+P_{2}\right) \\
& +\epsilon_{3}^{2}(25 / 12)\left(2 P_{2}^{2}-P_{2}+1\right) \\
& +2 \epsilon_{2} \epsilon_{3}(10 / 3) \zeta_{2}\left(1+P_{2}\right)+\epsilon_{4}^{2}\left(9 P_{4}\right)^{2} \\
& +\epsilon_{5}^{2}(5 / 14)\left(P_{2}^{2}+10 P_{2}+25\right)+90 \epsilon_{1} \epsilon_{4} P_{2} P_{4} .
\end{aligned}
$$

The Legendre polynomials occurring here depend on $\boldsymbol{u}_{1} \cdot \boldsymbol{u}_{2}$. Furthermore, the relations $\left\langle P_{2}\right\rangle_{0}=\left\langle P_{4}\right\rangle_{0}=$ $\left\langle P_{2} P_{4}\right\rangle_{0}=0,\left\langle P_{2}^{2}\right\rangle_{0}=1 / 5,\left\langle P_{4}^{2}\right\rangle_{0}=1 / 9,\left\langle P_{2}^{3}\right\rangle_{0}$ $=2 / 35,\left\langle P_{4} P_{2}^{2}\right\rangle_{0}=2 / 35,\left\langle P_{2} P_{4}^{2}\right\rangle_{0}=20 / 693$, and $\left\langle P_{4}^{3}\right\rangle_{0}=18 / 1001$ are used to obtain the expressions (46) and (48). Notice that $P_{2}^{2}=(18 / 35) \mathrm{P}_{4}+(2 / 7) \mathrm{P}_{2}$ $+1 / 5$. 
[1] S. Hess, Physica A 267, 58 (1999).

[2] J.D. Weeks, D. Chandler, and H.C. Andersen, J. Chem. Phys. 54, 5237 (1971).

[3] S. Hess, M. Kröger, and H. Voigt, Physica A 250, 58 (1998).

[4] F. Carnahan and K. E. Starling, J. Chem. Phys. 51, 635 (1969).

[5] L. Onsager, Phys.Rev. 62, 558 (1942); Ann. N. Y. Acad. Sci. 51, 627 (1949).

[6] M. A. Cotter, J. Chem. Phys. 67, 4268 (1976).

[7] A. Stroobants, H. N. W. Lekkerkerker, and D. Frenkel, Phys. Rev. Lett. 57, 1452 (1986); Phys. Rev. A 36, 2929 (1987).

[8] D. Baalss and S. Hess, Phys. Rev. Lett. 57, 86 (1986); Z. Naturforsch. 43 a, 662 (1988).

[9] D. Frenkel, J. Chem. Phys. 43, 4334 1991; J. A. C. Veerman and D. Frenkel Phys. Rev. A 41, 3237 (1990); Phys. Rev. A 41,3237 (1990).

[10] S. Hess, D. Frenkel, and M. P. Allen, Mol. Phys. 74, 765 (1991).

[11] S. C. McGrother, D. C. Williamson, and G. Jackson, J. Chem. Phys. 104, 6755 (1996).

[12] J. B. Gay and B. J. Berne, J.Chem.Phys. 74, 3316 (1981).

[13] D. J. Adams, G. R. Luckhurst, and R. W. Phippen, Mol. Phys. 61, 1575 (1987); E. de Miguel, L. F. Rull, M. K. Chalam, and K. E. Gubbins, Mol. Phys. 71, 1223 (1991); 72, 593 (1991); 74, 405 (1991); E. de Miguel, E. Martin del Rio, J. T. Brown, and M. P. Allen, J. Chem. Phys. 105, 4234 (1996); E. de Miguel and E. Martin del Rio, Phys. Rev. E 55, 2916 (1997).

[14] T. Gruhn and M. Schoen, Phys. Rev. E 55, 2861 (1997); Mol. Phys. 93, 681 (1998); J. Chem. Phys. 108, 9124 (1998).

[15] S. Hess, M. Kröger, W. Loose, C. Pereira Borgmeyer, R. Schramek, H. Voigt, and T. Weider, Simple and Complex Fluids under Shear, in: Monte Carlo and Molecular Dynamics of Condensed Matter Systems eds. K. Binder and G. Ciccotti, IPS Conf. Proc. 49, Bologna 1996, p. 825-841.

[16] P. A. Lebwohl and G. Lasher, Phys. Rev. A 5, 1350 (1972); G. Lasher, Phys. Rev. A 6, 426 (1972).

[17] T. Gruhn and S. Hess, Z.Naturforsch. 51 a, 1 (1996).

[18] S. Romano, Int. J. Mod. Phys. B 12, 2305 (1998); G. R. Luckhurst and S. Romano, Liq. Cryst. 26, 871 (1999).

[19] W. G. Hoover Molecular Dynamics, Springer, Berlin 1986; Computational Statistical Mechanics, Elsevier, Amsterdam 1991; Physica A 194, 450 (1993).

[20] M. P. Allen and D. J. Tildesley, Computer Simulation of Liquids, Clarendon, Oxford 1987.

[21] D. J. Evans and G. P. Morris, Statistical Mechanics of Nonequilibrium Liquids, Academic Press, London 1990.
[22] S. Hess and W. Loose, Molecular Dynamics: Test of Microscopic Models for the Material Properties of Matter, in: Constitutive laws and microstructure, eds. D. Axelrad and W. Muschik. Springer, Berlin 1988, p. 92; S. Hess, D. Baalss, O. Hess, W. Loose, J. F. Schwarzl, U. Stottut, and T. Weider in: Continuum models and discrete systems, ed. G. A. Maugin, Longman, Essex 1990, vol. 1, p. 18-30; T. Weider, U. Stottut, W. Loose, and S. Hess, Physica A 174, 1 (1991).

[23] S. Hess, Rheology and shear induced structure of fluids in: Rheological Modelling: Thermodynamical and Statistical Approaches, eds. J. Casas-Vázques and D. Jou, Lecture Notes in Physics 381, Springer, Berlin 1991, p. 51-73; Physikal. Blätter 44, 325 (1988).

[24] S. Hess, Constraints in Molecular Dynamics, Nonequilibrium Processes in Fluids via Computer Simulations, in: Computational Physics, eds. K. H. Hoffmann and M. Schreiber, Springer, Berlin 1996, p. 268-293.

[25] R. Haberlandt, S. Fritzsche, G. Peinel und K. Heinzinger, Molekular-Dynamik, Vieweg, Braunschweig 1995.

[26] A. J. Stone, Mol. Phys. 36, 241 (1978).

[27] S. Hess and L. Waldmann, Z.Naturforsch. 21 a, 1529 (1966); S. Hess and W. E. Köhler, Z.Naturforsch. 23 a, 1903 (1968).

[28] I. Pardowitz and S. Hess, Physica A 100, 540 (1980).

[29] I. Pardowitz and S. Hess, J. Chem. Phys. 76, 1485 (1982); M. Osipov and S. Hess, Mol. Phys. 78, 1191 (1993).

[30] H. Ehrentraut and W. Muschik, ARI 51, 149 (1998).

[31] W. Maier and A. Saupe, Z. Naturforsch. 14 a, 882 (1959); 15 a, 287 (1960).

[32] C. Pereira Borgmeyer, Zur Theorie der Strömungsausrichtung und Anisotropie der Viskosität in Flüssigkristallen, Diplomarbeit, TU-Berlin 1995.

[33] L. Bennett, A theoretical investigation of the nonequilibrium properties of liquid crystal, $\mathrm{PhD}$-thesis, TU-Berlin 1998; W\&T Verlag, Berlin 1999.

[34] J. P. Hansen and I. R. McDonald, Theory of Simple Liquids, (Academic Press, London 1986).

[35] J. A. Barker and D. Henderson, J. Chem. Phys. 47, 4714 (1967).

[36] J. A. Barker, J. Chem. Phys. 19, 1430 (1951); J. A. Pople, Proc. Roy. Soc. A 215, 67 (1952); A 219, 367 (1953); A 221, 498, 508 (1954).

[37] C. G. Gray and K. E. Gubbins, Theory of molecular fluids, vol. 1, Fundamentals, Clarendon Press, Oxford 1984.

[38] M. A. Osipov and S. Hess, J. Chem. Phys. 99, 4181 (1993). 